\title{
Antioxidative Effects of Nano-curcumin on Liver Mi- tochondria Function in Paraquat-induced Oxidative Stress
}

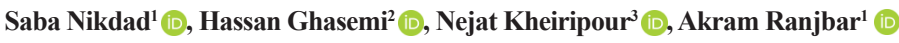 \\ 1. Medicinal Plants and Natural Products Research Center, Hamadan University of Medical Sciences, Hamadan, Iran. \\ 2. Department of Clinical Biochemistry, Abadan Faculty of Medical Sciences, Abadan, Iran. \\ 3. Research Center for Biochemistry and Nutrition in Metabolic Disorders, Kashan University of Medical Sciences, Kashan, Iran.
}

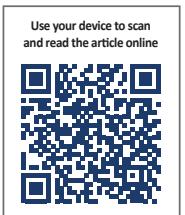

Citation Nikdad S, Ghasemi H, Kheiripour N, Ranjbar A. Antioxidative Effects of Nano-curcumin on Liver Mitochondria Function in Paraquat-induced Oxidative Stress. Research in Molecular Medicine. 2020; 8(1):37-42. https://doi.org/10.32598/rmm.8.1.37

doif https://doi.org/10.32598/rmm.8.1.37

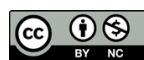

Article Type:

Research Article

Article info:

Received: 11 Dec 2019

Revised: 10 Jan 2020

Accepted: 27 Jan 2020

Keywords:

Nano-curcumin,

Curcumin, Paraquat,

Mitochondria, Liver

\begin{abstract}
A B S T R A C T
Background: Paraquat (PQ), as the most widely used herbicide in agriculture, induces poisoning in humans and animals mainly through oxidative stress. This study aimed to investigate the protective effects of nano-curcumin compared with curcumin against PQ-induced mitochondrial dysfunction.

Materials and Methods: Thirty-six Wistar rats were used in this study. They were separated into 6 groups: control subjects and animals poisoned with PQ that received treatment with or without curcumin and nano-curcumin for 7 days. Liver mitochondria were isolated, and oxidative stress markers, including Total Antioxidant Capacity (TAC), Lipid Peroxidation (LPO), Catalase (CAT) and Superoxide Dismutase (SOD) activity, as well as viability and mitochondrial membrane potential, were assessed.

Results: Poisoning by PQ significantly increased the LPO levels and considerably decreased the TAC, CAT, and SOD activity compared with the control subjects $(\mathrm{P}<0.05)$. PQ-induced oxidative significantly damaged the viability and membrane potential of mitochondrial compared with controls $(\mathrm{P}<0.05)$. Administration of nano-curcumin significantly increased the SOD activity, as well as viability and mitochondrial membrane potential in the $\mathrm{PQ}$ group $(\mathrm{P}<0.05)$. Besides, treatment by nano-curcumin in the $\mathrm{PQ}$ group significantly improved the lipid peroxidation process $(\mathrm{P}<0.05)$.
\end{abstract}

Conclusion: Nanocurcumin is more effective than curcumin in reducing the PQ-induced oxidative damage.

\section{Introduction}

oisoning with Paraquat $(\mathrm{PQ})$ is one of the most dangerous poisonings physicians face in the emergency wards. However, $\mathrm{PQ}$ is widely used in agriculture due to its herbicidal effect and low cost. PQ poisoning damages the cell membrane by generating free radicals and exerts adverse effects on body organs [1]. PQ can be absorbed through mouth or skin, and with massive exposure, it can kill humans within 3.5 hours.

* Corresponding Author:

Akram Ranjbar, PhD.

Address: Medicinal Plants and Natural Products Research Center, Hamadan University of Medical Sciences, Hamadan, Iran.

Phone: +98 (813) 8380031

E-mail: akranjbar2015@gmail.com 
The PQ toxicity in humans and animals appears as acute and chronic. Respiratory failure is the leading cause of death and causes disability and damage to the liver, kidney, heart, adrenal glands, spleen, and central nerves. It can also affect systems and organs like the central nervous system, liver, kidney, eye, and immune system. Accordingly, this chemical can endanger the health of humans, animals, and even aquatic organisms [2]. PQ toxicity is associated with the oxidation system and mitochondrial restoration. It seems that PQ-induced lung injury involves the production of $\mathrm{PQ}$ cation radicals from redox cycling. Furthermore, in PQ toxicity, the Release of Reactive Oxygen Species (ROS) such as superoxide anion, considerably decrease the levels of vital reducing equivalents such as NADPH (Nicotinamide adenine dinucleotide phosphate) and reduced glutathione [3].

None of the antidotes used in PQ poisoning have been clinically fully effective [4]. Treatments sometimes include clinically and, in some cases, empirically tested chemicals, including corticosteroids, immunosuppressive agents, fibrinolytic agents, colchicine and radiotherapy, iron-chelating by chelating agents like deferoxamine, selenium, exogenous glutathione and $\mathrm{N}$-acetyl cysteine $[2,5]$.

Antioxidants are chemicals that prevent the absorption of ROS [6]. Curcumin or diferuloylmethane is a biologically active ingredient of Curcuma longa from the ginger family. It is extracted from the rhizome of this plant, which is traditionally and commonly cultivated in Iran [7]. Different studies have documented the potential therapeutic effects of curcumin in a wide range of diseases like cancer, lung disease, neurodegenerative diseases, kidney disease, metabolites, heart disease, and other inflammatory diseases.

Curcumin has anti-oxidative, anti-inflammatory, and anti-cancer effects [8]. Moreover, curcumin shows a high ability to scavenge free radicals, exerts anti-inflammatory effects, and inhibits tumor growth. In cancerous cells, curcumin inhibits growth factors related to signaling pathways such as extracellular kinases and protein kinase C [9]. Curcumin can exert antioxidant activity by intensifying glutathione synthesis in poisoning [10].

Many approaches have been examined to increase the bioavailability of curcumin. These techniques include using adjuvants like piperine, liposomal curcumin, structural curcumin analogs such as 3,5-bis[(2-fluorophenyl) methylene]-4-piperidinone, curcumin phospholipid complex, and curcumin nanoparticles [11]. Some studies have shown that nanotechnology and encapsulation of curcumin in nano-emulsions (nano-curcumin) can enhance the medical properties of this material $[7,12]$. The current study aimed to evaluate the effects of nano-curcumin compared with curcumin against the PQ-induced liver mitochondrial dysfunction.

\section{Materials and Methods}

\section{Experimental Protocols}

In this study, 36 adult Wistar rats weighing 250-280 g were randomly divided into 6 following groups: normal saline, $5 \mathrm{mg} / \mathrm{kg}$ PQ [7], $100 \mathrm{mg} / \mathrm{kg}$ curcumin, $100 \mathrm{mg} /$ $\mathrm{kg}$ nano-curcumin, curcumin $+5 \mathrm{mg} / \mathrm{kg} \mathrm{PQ}$, and nanocurcumin $+5 \mathrm{mg} / \mathrm{kg}$ PQ.

\section{Preparation of liver mitochondria}

The liver was cut up with a scalpel in a cold mannitol solution containing $0.225 \mathrm{M}$ D-mannitol, $75 \mathrm{mM}$ sucrose, and $0.2 \mathrm{mM}$ Ethylenediaminetetraacetic acid (EDTA). The pieces of livers (30 g) were gently homogenized in a glass homogenizer with a Teflon pestle and then centrifuged at $700 \times \mathrm{g}$ for $10 \mathrm{~min}$ at $4^{\circ} \mathrm{C}$ to remove its nuclei, intact cells, and other non-subcellular components. The supernatants were collected in another falcon and were again centrifuged at $7000 \times \mathrm{g}$ for $20 \mathrm{~min}$. The supernatant contained crude microsomal fraction, and the pale, loose upper layer was rich in swollen or broken mitochondria, lysosomes, and some microsomes that were washed away from sediments [13].

\section{Total protein measurement}

Total protein concentration was measured using Bradford reagent and BSA standard. To prepare Bradford reagent, $100 \mathrm{mg}$ of Coomassie Brilliant Blue G-250 was dissolved in $50 \mathrm{~mL}$ of $95 \%$ ethanol and added to $100 \mathrm{~mL}$ of $85 \%$ phosphoric acid. Protein contents of the samples were read at $595 \mathrm{~nm}$ by adding $10 \mu \mathrm{L}$ of sample to $5 \mathrm{~mL}$ of reagent against the standard [14].

\section{Total Antioxidant Capacity (TAC) level measure-} ment

Total Antioxidant Capacity (TAC) was measured according to the FRAP method [15]. Briefly, in this method, the antioxidant compounds can be measured at low $\mathrm{pH}$ by reduction of Fe III complex ([3, 5-bis([2-fluorophenyl] methylene)-4-piperidinone] TPTZ) to ferrous (Fe II) (with bluish color) by measuring the absorption changes at $593 \mathrm{~nm}$ wavelength [15]. 


\section{Measuring Lipid Peroxidation (LPO) level}

Lipid peroxidation was assayed with the Yagi method. This method is based on the reaction between Malondialdehyde (MDA) - as the final product of lipid peroxidation - and Thiobarbituric Acid (TBA), which produces a red complex with optimum absorbance at $532 \mathrm{~nm}$. The standard curve was prepared by tetraethoxypropane solution [16].

\section{Measuring catalase (CAT) activity}

The activity of Catalase (CAT) was measured with Aeibi et al. approach followed by hydrogen peroxide $\left(\mathrm{H}_{2} \mathrm{O}_{2}\right)$ decomposition at $240 \mathrm{~nm}$. The reaction started by adding $30 \mathrm{mM} \mathrm{H}_{2} \mathrm{O}_{2}$ to a suitable volume of its mitochondria in $50 \mathrm{mM}$ sodium phosphate buffer at $\mathrm{pH}$ 7. The absorbance was then measured for $3 \mathrm{~min}$ at $240 \mathrm{~nm}$, and specific activity was calculated in units per mg protein [17].

\section{Measuring Superoxide Dismutase (SOD) activity}

To measure Superoxide Dismutase (SOD) activity, 20 $\mu \mathrm{L}$ mitochondria was extracted with $0.1 \mathrm{mM}$ EDTA in $0.3 \mathrm{mM}$ sodium cyanide and $3 \mathrm{mM}$ nitrobutetrazolium in a cuvette and stirred at $37^{\circ} \mathrm{C}$ for $5 \mathrm{~min}$. Then, $0.12 \mathrm{mM}$ riboflavin in $0.026 \mathrm{mM}$ phosphate buffer with $\mathrm{pH}=7.8$ was placed at room temperature for $10 \mathrm{~min}$. The absorbance was read at $560 \mathrm{~nm}$ for $5 \mathrm{~min}$, and the specific activity was determined in units per mg protein [18].

\section{Measuring of mitochondria viability}

The viability of mitochondria was evaluated by the MTT method. This method is based on the reduction of tetrazolium salt to purple formazan by the mitochondrial dehydrogenase enzyme in viable cells. The purple-colored formazan was measured at $560 \mathrm{~nm}$, and the reference wavelength of $630 \mathrm{~nm}$ with ELISA reader [19].

\section{Measuring mitochondrial membrane potential}

Rhodamine B is an unusual rhodamine derivative. Besides emitting red fluorescence when excited at the standard rhodamine excitation wavelength of $546 \mathrm{~nm}$ [20], it emits a green fluorescence similar to that typically associated with fluorescein compounds when it is excited at a wavelength of $485 \mathrm{~nm}$.

\section{Statistical analysis}

The study analysis was done in SPSS V. 16 and GraphPad Prism version 6.0 (GraphPad Software, San DiegoUSA). Statistical analysis comprised a 1-way Analysis of Variance (ANOVA) followed by post hoc Tukey test. Shapiro-Wilk test was used to examine the groups' normal distribution status. Results were expressed as the Mean \pm SD. $P<0.05$ indicates a statistically significant difference between groups.

\section{Results}

\section{LPO levels of liver mitochondria}

As shown in Figure 1, LPO in isolated mitochondria of liver tissue was significantly higher in the PQ group compared to the control group $(\mathrm{P}<0.05)$. Nano-curcumin treatment attenuates the LPO in isolated liver-mitochondria compared with the $\mathrm{PQ}$ group $(\mathrm{P}<0.05)$ (Figure 1).

\section{TAC of liver mitochondria}

Poisoning by PQ significantly decreased the TAC levels compared with the control subjects $(\mathrm{P}<0.01)$ (Figure 2 ).

\section{The CAT activity of liver mitochondria}

Our findings demonstrated that the level of CAT activity of mitochondria isolated from liver tissue in the $\mathrm{PQ}$ group significantly decreased compared with the control group $(\mathrm{P}<0.01)$. Treatment with curcumin and nano-curcumin did not significantly change the rate of CAT compared to the PQ group, and there were no significant differences with the healthy control group, which shows that they had normalized the CAT activity ( $\mathrm{P}>0.05)$ (Figure 3 ).

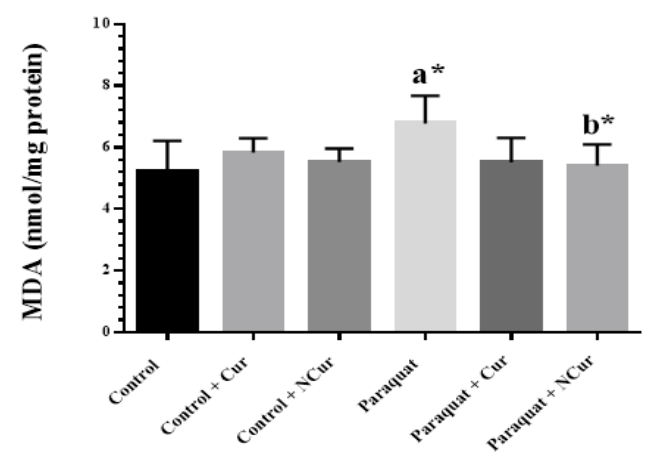

Figure 1. Lipid peroxidation level in liver mitochondria Data are presented as Mean \pm SD.

Cur: curcumin; NCur, nano-curcumin; a. significance compared with the healthy control group; b. significance compared with the PQ-poisoned control group.

$* \mathrm{P}<0.05 ; * * \mathrm{P}<0.01$ 


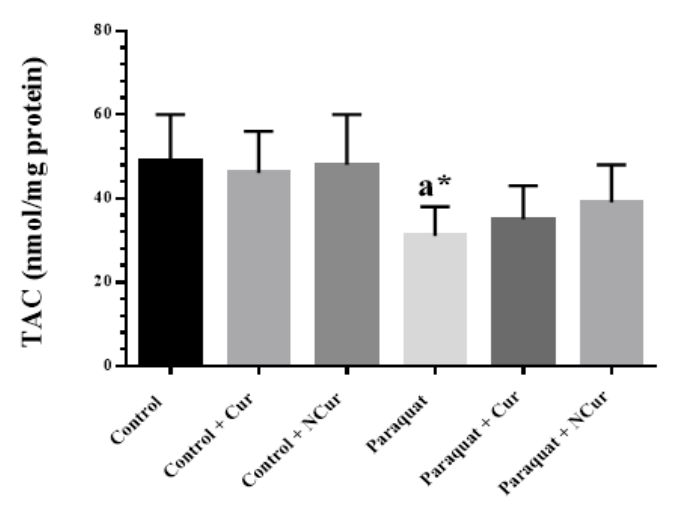

brmm

Figure 2. Total antioxidant capacity level in liver mitochondria Data are presented as Mean \pm SD.

Cur: curcumin; NCur: nano-curcumin; a. significance compared with the healthy control group; b. significance compared with the PQ-poisoned control group.

$* \mathrm{P}<0.05 ; * * \mathrm{P}<0.01$.

\section{The SOD activity of liver mitochondria}

The results showed that the level of SOD activity of mitochondria isolated from liver tissue in the PQ group was significantly reduced as compared with the healthy control group $(\mathrm{P}<0.05)$. Treatment with nano-curcumin and PQ increased SOD activity compared with the PQ group $(\mathrm{P}<0.05)$, but no significant differences were seen between the treatment with curcumin and the control group, which shows that catalase enzyme activity has reached the normal level (Figure 4).

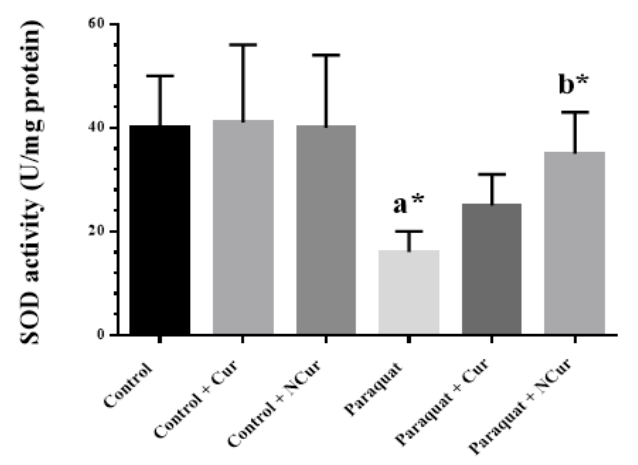

Brmm

Figure 4. Superoxide dismutase activity in liver mitochondria Data are presented as Mean \pm SD.

Cur: curcumin; NCur: nano-curcumin; a. significance compared with the healthy control group; b. significance compared with the $\mathrm{PQ}-$ poisoned control group.

$* \mathrm{P}<0.05 ; * * \mathrm{P}<0.01$.

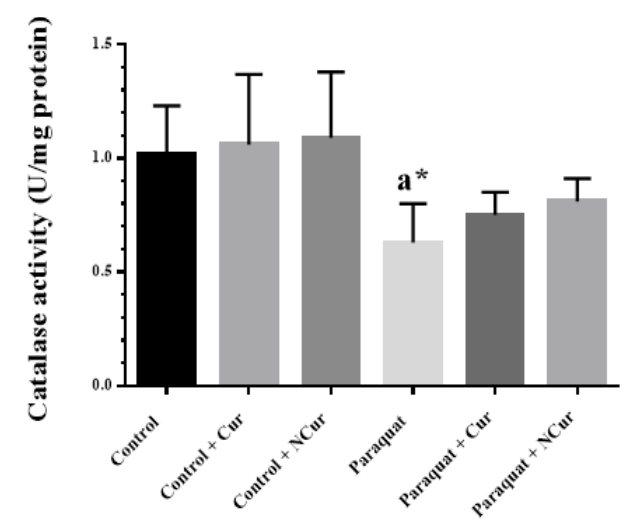

Figure 3. Catalase activity in liver mitochondria Data are presented as Mean \pm SD.

Cur: curcumin; NCur: nano-curcumin; a. significance compared with the healthy control group; $b$. significance compared with the PQ-poisoned control group.

${ }^{*} \mathrm{P}<0.05$; ** $\mathrm{P}<0.01$.

\section{The viability of liver mitochondria}

As shown in Figure 5, the viability of mitochondria isolated from liver tissue in the PQ group was significantly lower than that in the control group $(\mathrm{P}<0.001)$. Treatment with nano-curcumin improved the viability of isolated mitochondria of liver tissue as compared with the PQ group $(\mathrm{P}<0.05)$ (Figure 5).

The findings showed that the mitochondrial membrane potential significantly decreased in the PQ group compared with the control group $(\mathrm{P}<0.001)$. Treatment with nano-curcumin improved the membrane potential of mi-

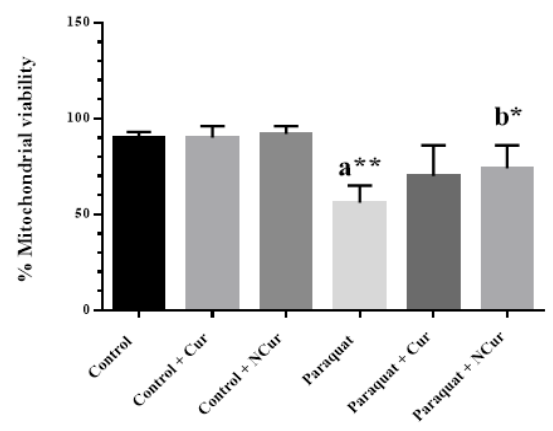

grmm

Figure 5. Viability of isolated liver mitochondria

Data are presented as Mean \pm SD.

Cur: curcumin; NCur: nano-curcumin; a. significance compared with the healthy control group; b. significance compared with the $\mathrm{PQ}$-poisoned control group.

${ }^{*} \mathrm{P}<0.05 ;{ }^{* *} \mathrm{P}<0.01$. 


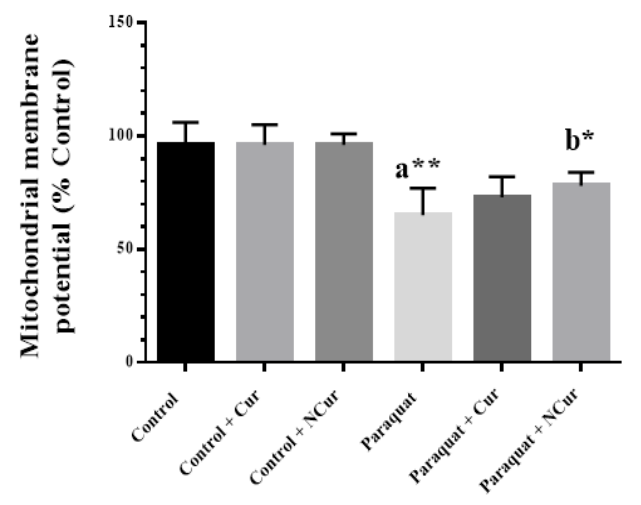

क $\mathrm{Rmm}$

Figure 6. Membrane potential of isolated liver mitochondria Data are presented as Mean \pm SD.

Cur: curcumin; NCur: nano-curcumin; a. significance compared with the healthy control group; $b$. significance compared with the PQ-poisoned control group.

${ }^{*} \mathrm{P}<0.05 ;{ }^{* *} \mathrm{P}<0.01$.

tochondria isolated from liver tissue as compared with the $\mathrm{PQ}$ group $(\mathrm{P}<0.05)$ (Figure 6).

\section{Discussion}

PQ-induced lung injury is characterized by fibrosis, pulmonary edema, and respiratory failure. The biochemical mechanism of the injury is complex and unclear; however, ROS may play a crucial role in mediating the cascade of biochemical changes induced by PQ. Accordingly, oxidative stress is an essential cause of PQ-induced lung injury [7]. The present study aimed to compare the effects of nano-curcumin and curcumin on the biological activity and membrane potential of mitochondria isolated from liver tissue in PQ subacute poisoning of male rats. The results showed the toxic effect of $P Q$ in the liver mitochondria through induction of toxic oxidative stress as supported by increasing LPO and decreasing TAC, SOD, and CAT activity and mitochondrial dysfunction.

The results showed that nano-curcumin has more antioxidant effects as compared with curcumin. According to the results of this study, it seems that nano-curcumin co-administration with PQ has better effects on biochemical parameters compared with curcumin. Curcumin encapsulation in nanoparticles makes it easier to enter into the cells and enhance the first line of antioxidant defense against oxidative damages due to toxins such as PQ. Han et al. reported that the rats' administration of PQ showed structural damage and liver tissue changes.
Also, PQ led to oxidative stress, increased expression of cytochrome P450 3A2 mRNA and mitochondrial damage, including mitochondrial membrane swelling, decreased mitochondrial cytochrome $\mathrm{C}$, and increased levels of apoptosis-induced proteins in the cell [21]. Our results showed that PQ induces oxidative damage in liver mitochondria. Our previous study showed that curcumin and nano-curcumin improved liver mitochondrial function mitochondrial dysfunction in aluminum phosphide toxicity [22].

Based on our study results, nano-curcumin as an effective antioxidant is responsible for neutralizing or scavenging the free radicals and thus inhibiting the toxic oxidative stress. In summary, our data disclosed that exposure to PQ induced remarkable oxidative toxicity on liver mitochondria. Improvement in oxidative stress factors using nano-curcumin, suggest that nano-curcumin treatment could protect the liver against the oxidative injuries of PQ by scavenging ROS and stabilizing the oxidative status.

\section{Ethical Considerations}

\section{Compliance with ethical guidelines}

The Ethics Committee of Hamadan University of Medical Sciences approved this study (IR.UMSHA. REC.1397.281).

\section{Funding}

This study was supported by the Vice-Chancellor of Research of Hamadan University of Medical Sciences (Grant number: 9704262218).

\section{Authors contribution's}

Review, comment and approve of the final draft: All authors; Performing the experiment: Saba Nikdad, Nejat Kheiripour; data analysis and co-wrote the paper: Hassan Ghasemi; Supervision: Akram Ranjbar.

\section{Conflict of interest}

The authors declared no conflict of interest.

\section{Acknowledgements}

The authors would like to thank Research Affairs of Medicinal Plants and Natural Products Research Center, Hamadan University of Medical Sciences. 


\section{References}

[1] Vale JA, Meredith TJ, Buckley BM. Paraquat poisoning: Clinical features and immediate general management. Hum Exp Toxicol. 1987; 6(1):41-7. [DOI:10.1177/096032718700600107] [PMID]

[2] Goel A, Singh O. Herbicides poisoning: Paraquat and diquat. In: Singh O, Juneja D. Principles and Practice of Critical Care Toxicology. New Delhi: JP Medical Ltd; 2019. https:/ / books.google.com/books?id=m3KSDwAAQBAJ\&dq

[3] Ranjbar A, Pasalar P, Sedighi AR, Abdollahi M. Induction of oxidative stress in paraquat formulating workers. Toxicol Lett. 2002; 131(3):191-4. [DOI:10.1016/S0378-4274(02)000334]

[4] Yoon SC. Clinical outcome of paraquat poisoning. Korean J Intern Med. 2009; 24(2):93-4. [DOI:10.3904/kjim.2009.24.2.93] [PMID] [PMCID]

[5] Gawarammana IB, Buckley NA. Medical management of paraquat ingestion. Br J Clin Pharmacol. 2011; 72(5):745-57. [DOI:10.1111/j.1365-2125.2011.04026.x] [PMID] [PMCID]

[6] Ranjbar A. Evidence of oxidative damage in paraquat toxicity. Zahedan J Res Med Sci. 2014; 16(12):1-8. http://zjrms. com/articles/1089.html

[7] Hosseini A, Rasaie D, Soleymani Asl S, Nili Ahmadabadi A, Ranjbar A. Evaluation of the protective effects of curcumin and nanocurcumin against lung injury induced by sub-acute exposure to paraquat in rats. Toxin Rev. 2019 October. [DOI :10.1080/15569543.2019.1675707]

[8] Menon VP, Sudheer AR. Antioxidant and anti-inflammatory properties of curcumin. In: Aggarwal BB, Surh YJ, Shishodia S, editors. The Molecular Targets and Therapeutic Uses of Curcumin in Health and Disease, Advances in Experimental Medicine and Biology. Vol. 595. Boston, MA: Springer; 2007. [DOI:10.1007/978-0-387-46401-5_3] [PMID]

[9] Motterlini R, Foresti R, Bassi R, Green CJ. Curcumin, an antioxidant and anti-inflammatory agent, induces heme oxygenase-1 and protects endothelial cells against oxidative stress. Free Radic Biol Med. 2000; 28(8):1303-12. [DOI:10.1016/ S0891-5849(00)00294-X]

[10] Fu Y, Zheng S, Lin J, Ryerse J, Chen A. Curcumin protects the rat liver from CCl4-caused injury and fibrogenesis by attenuating oxidative stress and suppressing inflammation. Mol Pharmacol. 2008; 73(2):399-409. [DOI:10.1124/ mol.107.039818] [PMID]

[11] Anand P, Kunnumakkara AB, Newman RA, Aggarwal BB. Bioavailability of curcumin: Problems and promises. Mol Pharm. 2007; 4(6):807-18. [DOI:10.1021/mp700113r] [PMID]

[12] Flora G, Gupta D, Tiwari A. Nanocurcumin: A promising therapeutic advancement over native curcumin. Crit Rev Ther Drug Carrier Syst. 2013; 30(4):331-68. [DOI:10.1615/ CritRevTherDrugCarrierSyst.2013007236] [PMID]

[13] Ghazi-Khansari M, Mohammadi-Bardbori A, Hosseini MJ. Using Janus green B to study paraquat toxicity in rat liver mitochondria: Role of ACE inhibitors (thiol and nonthiol ACEi). Ann N Y Acad Sci. 2006; 1090(1):98-107. [DOI:10.1196/annals.1378.010] [PMID]
[14] Jones CG, Hare JD, Compton SJ. Measuring plant protein with the Bradford assay. J Chem Ecol. 1989; 15(3):979-92. [DOI:10.1007/BF01015193] [PMID]

[15] Benzie IFF, Strain JJ. The Ferric Reducing Ability of Plasma (FRAP) as a measure of "antioxidant power": The FRAP assay. Anal Biochem. 1996; 239(1):70-6. [DOI:10.1006/ abio.1996.0292] [PMID]

[16] Ohkawa H, Ohishi N, Yagi K. Assay for lipid peroxides in animal tissues by thiobarbituric acid reaction. Anal Biochem. 1979; 95(2):351-8. [DOI:10.1016/0003-2697(79)90738-3]

[17] Aebi H. Catalase. In: Bergmeyer HU, editor. Methods of Enzymatic Analysis. Vol. 2. Cambridge, MA: Academic Press; 1974. [DOI:10.1016/B978-0-12-091302-2.50032-3]

[18] Masayasu M, Hiroshi Y. A simplified assay method of superoxide dismutase activity for clinical use. Clin Chim Acta. 1979; 92(3):337-42. [DOI:10.1016/0009-8981(79)90211-0]

[19] Supino R. MTT assays. In: O'Hare S, Atterwill CK, editors. In Vitro Toxicity Testing Protocols, Methods in Molecular Biology ${ }^{\mathrm{TM}}$. Vol. 43. Totowa, NJ: Humana Press; 1995. [DOI:10.1385/0-89603-282-5:137] [PMID]

[20] Sakamuru S, Attene-Ramos MS, Xia M. Mitochondrial membrane potential assay. In: Zhu $\mathrm{H}, \mathrm{Xia} \mathrm{M}$, editors. In: High-Throughput Screening Assays in Toxicology, Methods in Molecular Biology. Vol. 1473. New York, NY: Humana Press; 2016. [DOI:10.1007/978-1-4939-6346-1_2] [PMID] [PMCID]

[21] Han J, Zhang Z, Yang Sh, Wang J, Yang X, Tan D. Betanin attenuates paraquat-induced liver toxicity through a mitochondrial pathway. Food Chem Toxicol. 2014; 70:100-6. [DOI:10.1016/j.fct.2014.04.038] [PMID]

[22] Ranjbar A, Gholami L, Ghasemi H, Kheiripour N. Eeffects of nano-curcumin and curcumin on the oxidant and antioxidant system of the liver mitochondria in aluminum phosphide-induced experimental toxicity. Nanomed J. 2020; 7(1):58-64. http://nmj.mums.ac.ir/article_13628.html 